\title{
An Extended Burr XII Distribution: Properties, Inference and Applications
}

\author{
Gauss M. Cordeiro \\ Departamento de Estatística \\ Universidade Federal de Pernambuco, Pernambuco, Brazil \\ gauss@de.ufpe.br
}

M. E. Mead

Department of Statistics, Mathematics and Insurance

Zagazig University, Egypt

mead9990@gmail.com

\author{
Ahmed Z. Afify \\ Department of Statistics, Mathematics and Insurance \\ Benha University, Egypt \\ ahmed.afify@fcom.bu.edu.eg \\ Adriano K. Suzuki \\ Departamento de Matemática Aplicada e Estatística \\ Universidade de São Paulo, São Paulo, Brazil \\ suzuki@icmc.usp.br \\ Amarat A. K. Abd El-Gaied \\ Department of Statistics, Mathematics and Insurance \\ Zagazig University, Egypt \\ amira.emad@yahoo.com
}

\begin{abstract}
We propose and study a new continuous model named the Marshall-Olkin exponentiated Burr XII (MOEBXII) distribution. It contains several special cases, namely the Marshall-Olkin exponentiated loglogistic, Marshall-Olkin exponentiated Lomax, Marshall-Olkin Burr XII, Marshall-Olkin log-logistic, Marshall-Olkin Lomax distributions, among others, and most importantly includes all four of the most common types of hazard function: monotonically increasing or decreasing, bathtub and arc-shaped hazard functions. Some of its structural properties are obtained such as the ordinary and incomplete moments, quantile and generating functions, order statistics and probability weighted moments. The maximum likelihood and least square methods are used to estimate the model parameters. A simulation study is performed to evaluate the precision of the estimates from both methods. The usefulness of the new model is illustrated by means of two real data sets.
\end{abstract}

Keywords: Exponentiated Burr XII, Hazard Function, Marshall-Olkin Family, Maximum Likelihood, Order Statistic, Rényi entropy.

\section{Introduction}

The Burr XII (BXII) distribution includes as sub-models the logistic and Weibull distributions and it is very popular for modeling lifetime data and phenomenon with different failure rates. It can fit a wide range of empirical data. Different values of its parameters cover a broad set of skewness and kurtosis. Hence, it is used in various fields 
such as finance, hydrology and reliability to model a variety of data types. Examples of data modeled by the BXII distribution are household income, crop prices, insurance risk, flood levels and failure data.

Recently, many generalizations of the BXII distribution have been proposed. For example, the beta BXII (Paranaba et al., 2011), Kumaraswamy BXII (Paranaba et al., 2013), beta exponentiated BXII (Mead, 2014), Marshall-Olkin BXII (Al-Saiarie et al., 2014), McDonald BXII (Gomes et al., 2015), Weibull BXII (Afify et al., 2016) and Kumaraswamy exponentiated BXII (Mead and Afify, 2017) distributions.

The cumulative distribution function (cdf) of the exponentiated Burr XII (EBXII) distribution is given by (for $x \geq 0$ )

$$
G(x ; c, k, \lambda)=\left[1-\left(1+x^{c}\right)^{-k}\right]^{\lambda},
$$

where $c, k$ and $\lambda$ are positive shape parameters.

The probability density function (pdf) of the EBXII becomes

$$
g(x ; c, k, \lambda)=\lambda c k x^{c-1}\left(1+x^{c}\right)^{-k-1}\left[1-\left(1+x^{c}\right)^{-k}\right]^{\lambda-1} .
$$

The procedure of adding one or two shape parameters to a class of distributions to obtain more flexibility is a well-known technique in the statistical literature. Marshall and Olkin (1997) proposed a method of adding a shape parameter into a family of distributions and many authors used their method to extend several well-known distributions in recent years. This family is called the Marshall-Olkin-G (MO-G) class. Tahir and Nadarajah (2015) listed 28 published papers on distributions in this class.

Consider a baseline distribution with cdf $G(x)$ and pdf $g(x)$. Thus, the cdf and pdf of the MO-G family are defined by (for $x \in \mathfrak{R}$ )

$$
F(x ; \alpha)=1-\frac{\alpha[1-G(x)]}{1-\bar{\alpha}[1-G(x)]}
$$

and

$$
f(x ; \alpha)=\frac{\alpha g(x)}{\{1-\bar{\alpha}[1-G(x)]\}^{2}}
$$

respectively, where $\alpha>0$ is a shape parameter and $\bar{\alpha}=1-\alpha$. For $\alpha=1$, we obtain the baseline distribution, i.e. $F(x ; 1)=G(x)$.

The hazard rate function (hrf) corresponding to (4) is given by

$$
h(x ; \alpha)=\frac{r(x)}{1-\bar{\alpha}[1-G(x)]},
$$

where $r(x)=g(x) /[1-G(x)]$ is the hrf of the parent distribution. It is clear from equation (5) that $h(x ; \alpha) / r(x)$ is increasing in $x$ for $\alpha \geq 1$ and decreasing in $x$ for $0<$ $\alpha \leq 1$. According to Marshall and Olkin, the shape parameter $\alpha$ is called "tilt parameter", since the hrf $h(x ; \alpha)$ of the generated distribution is shifted below $(\alpha \geq 1)$ or above $(0<\alpha \leq 1)$ from the baseline hrf, say $h_{G}(x)$. In fact, for all $x>0, h(x ; \alpha) \leq$ $r(x)$ when $\alpha \geq 1$, and $h(x ; \alpha) \geq r(x)$ when $0<\alpha \leq 1$. Further details were addressed by Marshall and Olkin (1997). 
In this paper, the EBXII distribution is embedded in a larger family by adding a new shape parameter. The model generated by applying the MO transformation to the EBXII distribution is called the Marshall-Olkin exponentiated Burr XII (MOEBXII) distribution. The new distribution has the advantage of being capable of modelling various shapes of aging and failure criteria. Further, the MOEBXII model is shown to provide better fits than at least seven other models each one having the same number of parameters. We hope that the proposed distribution will attract wider applications in reliability, engineering and other areas of research.

The rest of the paper is outlined as follows. In Section 2, we define the MOEBXII distribution, provide plots of the pdf and hrf, give some special models and derive a linear representation for the pdf. In Section 3, we obtain some mathematical properties including quantile function (qf), ordinary, central and incomplete moments, moment generating function (mgf), moments of the residual and reversed residual lifes, order statistics and probability weighted moments (PWMs). In Section 4, we discuss the maximum likelihood and the least square methods to estimate the model parameters. Two simulation studies are performed in Section 5 in order to verify the consistency of the estimates. In Section 6, we prove empirically the potentiality of the new distribution by means of two real data sets. Finally, some concluding remarks are offered in Section 7.

\section{The MOEBXII Distribution}

In this section, we define the MOEBXII distribution and provide its eleven sub-models. By inserting (1) in equation (3), the cdf of the MOEBXII distribution (for $x>0$ ), say $F(x)=F(x ; c, k, \lambda, \alpha)$, is given by

$$
F(x)=1-\frac{\alpha\left\{1-\left[1-\left(1+x^{c}\right)^{-k}\right]^{\lambda}\right\}}{1-\bar{\alpha}\left\{1-\left[1-\left(1+x^{c}\right)^{-k}\right]^{\lambda}\right\}},
$$

where $c, k, \lambda$ and $\alpha$ are positive shape parameters.

Its density function, say $f(x)=f(x ; c, k, \lambda, \alpha)$, becomes

$$
f(x)=\frac{\alpha \lambda c k x^{c-1}\left(1+x^{c}\right)^{-k-1}\left[1-\left(1+x^{c}\right)^{-k}\right]^{\lambda-1}}{\left(1-\bar{\alpha}\left\{1-\left[1-\left(1+x^{c}\right)^{-k}\right]^{\lambda}\right\}\right)^{2}} .
$$

Henceforth, let $X \sim \operatorname{MOEBXII}(c, k, \lambda, \alpha)$ be a random variable having the pdf (7).

The hrf and cumulative hazard rate function (chrf) of $X$ are given by

and

$$
h(x ; c, k, \lambda, \alpha)=\frac{r(x ; c, k, \lambda)}{1-\bar{\alpha}\left\{1-\left[1-\left(1+x^{c}\right)^{-k}\right]^{\lambda}\right\}}
$$

$$
H(x ; c, k, \lambda, \alpha)=\log \left(\frac{1-\bar{\alpha}\left\{1-\left[1-\left(1+x^{c}\right)^{-k}\right]^{\lambda}\right\}}{\alpha\left\{1-\left[1-\left(1+x^{c}\right)^{-k}\right]^{\lambda}\right\}}\right),
$$

respectively, where

$$
r(x ; c, k, \lambda)=\frac{\lambda c k x^{c-1}\left(1+x^{c}\right)^{-k-1}\left[1-\left(1+x^{c}\right)^{-k}\right]^{\lambda-1}}{1-\left[1-\left(1+x^{c}\right)^{-k}\right]^{\lambda}}
$$

is the hrf of the EBXII model. 
For $0<\alpha<1$, we provide a simple motivation for the MOEBXII distribution in the medical context as follows. Consider a random sample $W_{1}, \ldots, W_{N}$, where the $W_{i}$ 's are independent and identically distributed having the EBXII density (2). Let $N$ have a geometric random variable with probability mass function $(\mathrm{pmf}) P(N=k)=\alpha(1-$ $\alpha)^{k-1}$ (for $k \geq 1$ ). Then, the density of the random variable $X=\min \left(W_{1}, \ldots, W_{N}\right)$ is that of (7). For $\alpha>1$, we can use $\alpha^{-1}$ instead of $\alpha$ in the pmf of $N$. This setup is usually common in oncology, where $N$ represents the amount of cells with metastasis potential and $W_{i}$ denotes the time for the $i$ th cell to metastasize. So, $X$ represents the recurrence time of the cancer.

The MOEBXII distribution is a very flexible model that approaches to different distributions. Its eleven sub-models are listed in Table 1. Figure 1 displays some plots of the MOEBXII density for selected values of $c, k, \lambda$ and $\alpha$. These plots illustrate the versatility and modality of the new distribution. The plots in Figure 2 reveal that the hrf of $X$ can have bathtub, unimodal, increasing and decreasing shapes.

Table 1: Sub-models of the MOEBXII distribution

\begin{tabular}{|c|l|l|l|l|l|l|}
\hline No. & Distribution & $c$ & $k$ & $\lambda$ & $\alpha$ & Author \\
\hline 1 & MOBXII & $c$ & $k$ & 1 & $\alpha$ & Al-Saiarie et al. (2014) \\
\hline 2 & MOELo & 1 & $k$ & $\lambda$ & $\alpha$ & New \\
\hline 3 & MOELL & $c$ & 1 & $\lambda$ & $\alpha$ & New \\
\hline 4 & MOLo & 1 & $k$ & 1 & $\alpha$ & - \\
\hline 5 & MOLL & $c$ & 1 & 1 & $\alpha$ & - \\
\hline 6 & EBXII & $c$ & $k$ & $\lambda$ & 1 & \\
\hline 7 & ELo & 1 & $k$ & $\lambda$ & 1 & \\
\hline 8 & ELL & $c$ & 1 & $\lambda$ & 1 & \\
\hline 9 & BXII & $c$ & $k$ & 1 & 1 & \\
\hline 10 & Lo & 1 & $k$ & 1 & 1 & \\
\hline 11 & LL & $c$ & 1 & 1 & 1 & \\
\hline
\end{tabular}

Abbreviations: Lo=Lomax and LL=Log-Logistic. 

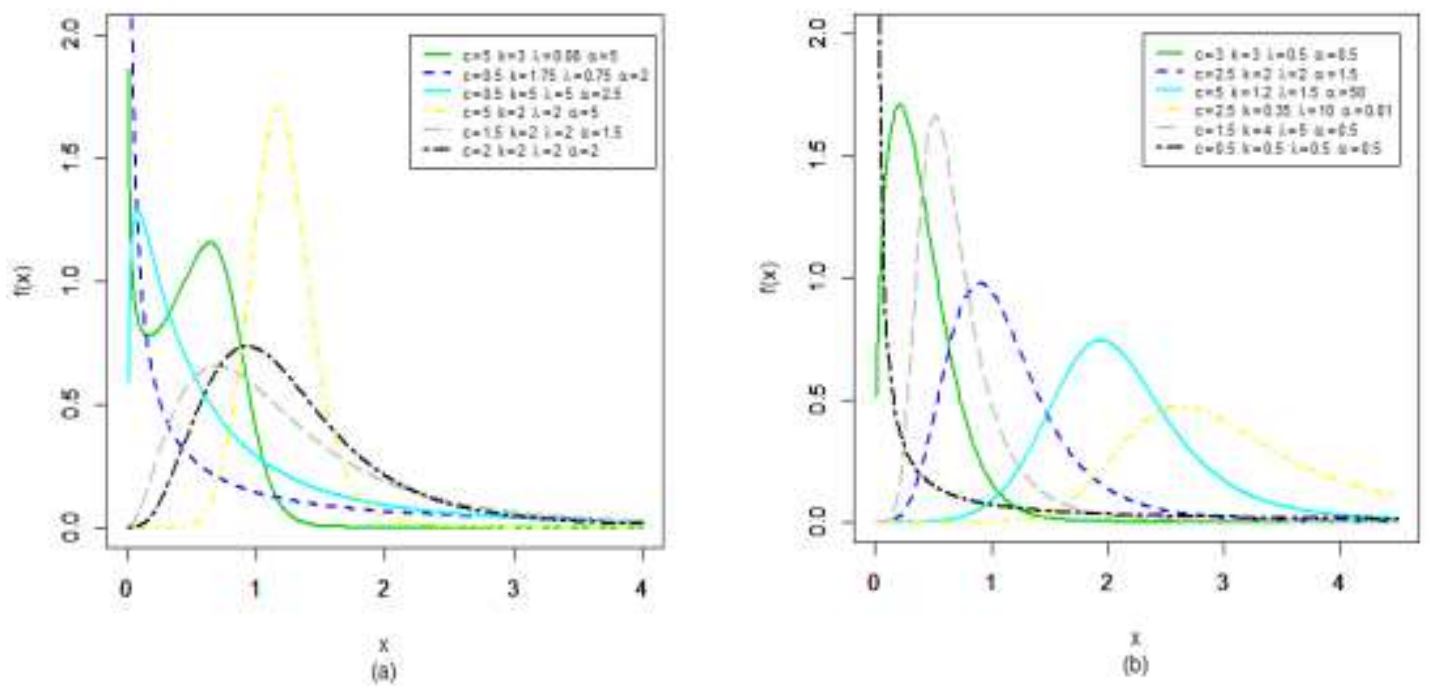

Figure 1: Plots of the MOEBXII density for selected parameter values.
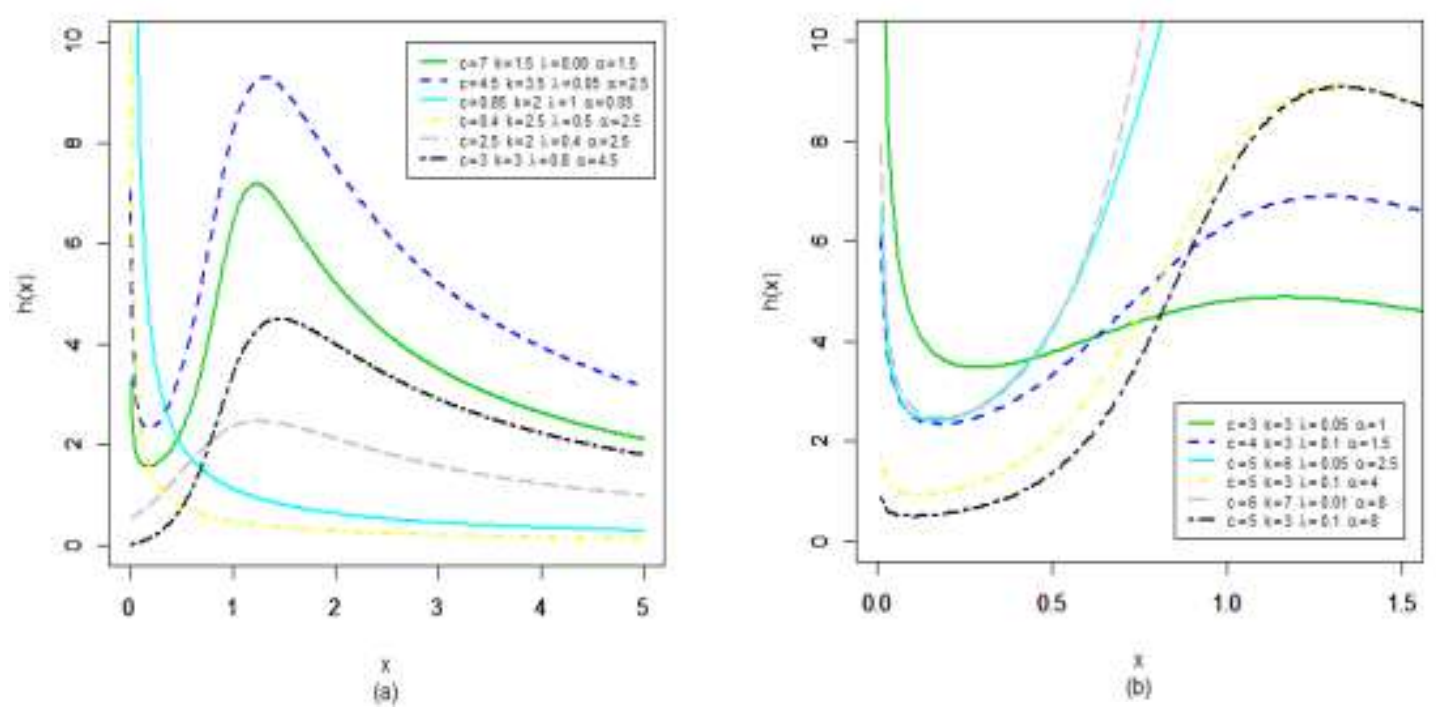

Figure 2: Plots of the MOEBXII hrf for selected parameter values.

\subsection{Linear Representation}

An expansion for equation (7) can be derived using the power series

$$
(1-z)^{-v}=\sum_{j=0}^{\infty} \frac{\Gamma(v+j)}{j ! \Gamma(v)} z^{j},
$$

where $|z|<1, v>0$ and $\Gamma(v)=\int_{0}^{\infty} t^{v-1} \mathrm{e}^{-t} \mathrm{~d} t$ is the gamma function. Based on the linear representation of Cordeiro et al. (2014) for the MO-G family and using (8) in (7) when $\alpha \in(0,1)$, we can write 


$$
\begin{array}{rl}
f(x)=\alpha \lambda c & k \sum_{i=0}^{\infty} \sum_{j=i}^{\infty}(-1)^{i}(j+1) \bar{\alpha}^{j}\left(\begin{array}{l}
j \\
i
\end{array}\right) \\
& \times x^{c-1}\left(1+x^{c}\right)^{-k-1}\left[1-\left(1+x^{c}\right)^{-k}\right]^{\lambda(i+1)-1} .
\end{array}
$$

Using the generalized binomial expansion, we obtain

$$
\begin{gathered}
f(x)=\alpha \sum_{i, m=0}^{\infty} \sum_{j=i}^{\infty}(-1)^{i+m}(j+1) \bar{\alpha}^{j}\left(\begin{array}{l}
j \\
i
\end{array}\right)\left(\begin{array}{c}
\lambda(i+1)-1 \\
m
\end{array}\right) \\
\times \lambda c k x^{c-1}\left(1+x^{c}\right)^{-k(m+1)-1} .
\end{gathered}
$$

Then, the pdf of $X$ can be expressed as a linear combination of BXII densities

$$
f(x)=\sum_{m=0}^{\infty} b_{m} g_{m+1}(x),
$$

where $g_{m+1}(x)$ is the pdf of the BXII model with shape parameters $c$ and $(m+1) k$ and (for $m \geq 0$ )

$$
b_{m}=\frac{\alpha \lambda}{m+1} \sum_{i=0}^{\infty} \sum_{j=i}^{\infty}(-1)^{i+m}(j+1) \bar{\alpha}^{j}\left(\begin{array}{l}
j \\
i
\end{array}\right)\left(\begin{array}{c}
\lambda(i+1)-1 \\
m
\end{array}\right) .
$$

Otherwise, if $\alpha>1$, we can rewrite (7), using (8) and following the same developments of Cordeiro et al. (2014), as

$$
f(x)=\alpha^{-1}\left(1-\alpha^{-1}\right) \lambda c k \sum_{j=0}^{\infty}(j+1) x^{c-1}\left(1+x^{c}\right)^{-k-1}\left[1-\left(1+x^{c}\right)^{-k}\right]^{\lambda(j+1)-1} \text {. }
$$

Applying the generalized binomial expansion gives

$$
\begin{aligned}
f(x)=\lambda \alpha^{-1} & \left(1-\alpha^{-1}\right) c k \sum_{j, m=0}^{\infty}(-1)^{m}(j+1) \\
& \times\left(\begin{array}{c}
\lambda(j+1)-1 \\
m
\end{array}\right) x^{c-1}\left(1+x^{c}\right)^{-k(m+1)-1} .
\end{aligned}
$$

Then, the pdf of $X$ when $\alpha>0$ can be expressed as a linear combination of BXII densities given by

$$
f(x)=\sum_{m=0}^{\infty} q_{m} g_{m+1}(x),
$$

where

$$
q_{m}=\frac{(-1)^{m} \lambda \alpha^{-1}\left(1-\alpha^{-1}\right)}{m+1} \sum_{j=0}^{\infty}(j+1)\left(\begin{array}{c}
\lambda(j+1)-1 \\
m
\end{array}\right) .
$$

Equations (9) and (10) have the same linear representation except for the coefficients. They are the main results of this section. So, some structural properties of the MOEBXII model can be determined from these equations and those properties of the BXII distribution. From now on, we work only with equation (9).

\section{Mathematical Properties}


In this section, we obtain some mathematical properties of the MOEBXII distribution including qf and random number generation, ordinary, central and incomplete moments, mgf and moments of the residual and reversed residual lifes.

Let $Z$ be a random variable having the two-parameter BXII density given by

$$
g(z ; \alpha, k)=c k z^{c-1}\left(1+z^{c}\right)^{-k-1},
$$

where $c$ and $k$ are positive shape parameters.

The $r$ th ordinary and incomplete moments of $Z$ are given (for $r<c k$ ) by

$$
\mu_{r, Z}^{\prime}=k B\left(k-\frac{r}{c}, \frac{r}{c}+1\right) \quad \text { and } \quad \varphi_{r, Z}(t)=k B\left(t^{c} ; k-\frac{r}{c}, \frac{r}{c}+1\right),
$$

respectively, where $B(a, b)=\int_{0}^{1} t^{a-1}(1-t)^{b-1} d t$ and $B(y ; a, b)=\int_{0}^{y} t^{a-1}(1-$ $t)^{b-1)} d t$ are the beta and incomplete beta functions, respectively.

\subsection{Moments}

The $r$ th ordinary moment of $X$ is given by

$$
\mu_{r}^{\prime}=E\left(X^{r}\right)=\sum_{m=0}^{\infty} b_{m} \int_{0}^{\infty} x^{r} g_{m+1}(x) d x
$$

For $r<k c$, we obtain

$$
\mu_{r}^{\prime}=k \sum_{m=0}^{\infty} b_{m}(m+1) B\left(k(m+1)-\frac{r}{c}, \frac{r}{c}+1\right) .
$$

The mean of $X$ follows by setting $r=1$ in (12) as

$$
\mu_{1}^{\prime}=k \sum_{m=0}^{\infty} b_{m}(m+1) B\left(k(m+1)-\frac{1}{c}, \frac{1}{c}+1\right) .
$$

The $s$ th central moment $\left(\mu_{s}\right)$ of $X$ is given by

$$
\mu_{s}=E(X-\mu)^{s}=\sum_{i=0}^{s}(-1)^{i}\left(\begin{array}{l}
s \\
i
\end{array}\right)\left(\mu_{1}^{\prime}\right)^{s} \mu_{s-i}^{\prime} .
$$

The skewness and kurtosis measures can be calculated from the central moments using well-known relationships.

The $s$ th incomplete moment of $X$ is given by $\varphi_{s}(t)=\int_{0}^{t} x^{s} f(x) d x$. It follows from equation (9)

$$
\varphi_{s}(t)=\sum_{m=0}^{\infty} b_{m} \int_{0}^{t} x^{s} g_{m+1}(x) d x,
$$

and then using the incomplete beta function, we obtain (for $s<k c$ )

$$
\varphi_{s}(t)=k \sum_{m=0}^{\infty}(m+1) b_{m} B\left(t^{c} ; k(m+1)-\frac{s}{c}, \frac{s}{c}+1\right) .
$$


The first incomplete moment of $X$ follows from the last equation by setting $s=1$. It can be applied to obtain mean deviations, Bonferroni and Lorenz curves, mean residual and waiting times and totality of deviations from the mean and median.

The effects of the parameter $\alpha$ on the mean, variance, skewness and kurtosis for given values of $\lambda, c$ and $k$ are displayed in Figures 3 and 4, respectively.

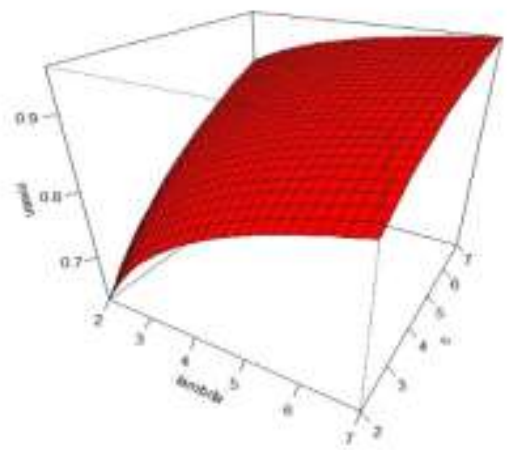

nam
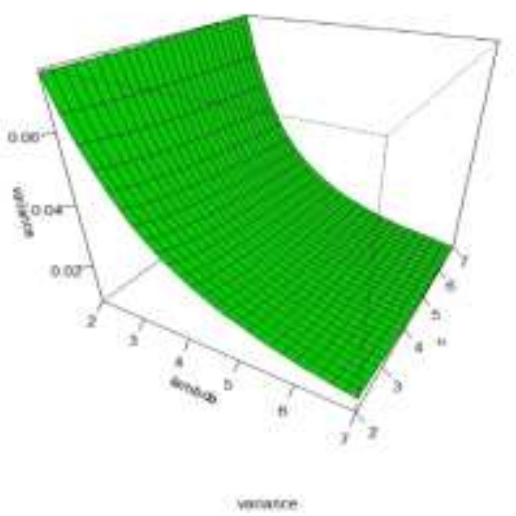

Figure 3: Plots of mean and variance of the MOEBXII distribution.

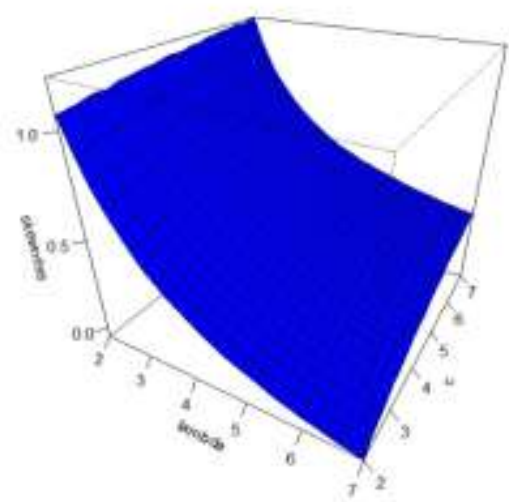

atimens

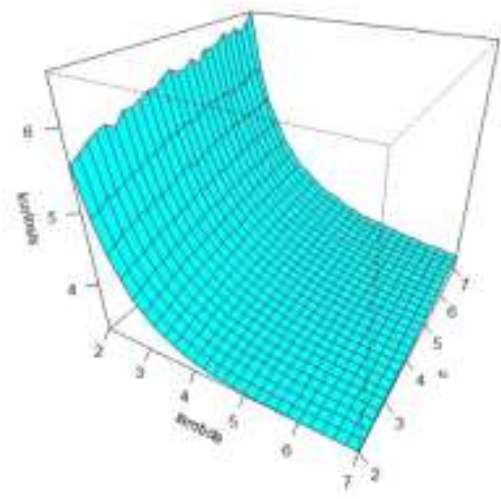

hartions

Figure 4: Plots of skewness and kurtosis of the MOEBXII distribution.

\subsection{Quantile and Generating Functions}

The qf of $X$ is obtained by inverting (6) as

$$
Q(u)=\left(\left\{1-\left[\frac{\alpha u}{1-(1-\alpha) u}\right]^{\frac{1}{\lambda}}\right\}^{\frac{-1}{k}}-1\right)^{\frac{1}{c}}, 0<u<1 .
$$

Setting $u=0.5$ in (13) gives the median of $X$. Simulating the MOEBXII random variable is straightforward. If $U$ is a uniform variate on the unit interval $(0,1)$, then the random variable $X=Q(U)$ follows (7). 
The mgf of $X$, say $M_{X}(t)=E\left(\mathrm{e}^{t X}\right)$, can be obtained from (9) as

$$
M_{X}(t)=\sum_{m=0}^{\infty} b_{m} M_{m+1}(t)
$$

where $M_{m+1}(t)$ is the mgf of the BXII distribution with parameters $c,(m+1) k$.

First, we provide the mgf of the two-parameter BXII distribution as defined by Afify et al. (2016). We can write the $\operatorname{mgf}$ of $Z$ with pdf (11), say $M_{Z}(t)$, (for $\left.t<0\right)$ as

$$
M_{Z}(t)=c k \int_{0}^{\infty} \mathrm{e}^{\mathrm{zt}} z^{c-1}\left(1+z^{c}\right)^{-k-1} d z .
$$

We require the Meijer G-function defined by

$$
G_{p, q}^{s, n}\left(\left.x\right|_{b_{1}, \ldots, b_{q}} ^{a_{1}, \ldots, a_{p}}\right)=\frac{1}{2 \pi i} \int_{L} \frac{\prod_{j=1}^{s} \Gamma\left(b_{j}+t\right) \prod_{j=1}^{n} \Gamma\left(1-a_{j}-t\right)}{\prod_{j=n+1}^{p} \Gamma\left(a_{j}+t\right) \prod_{j=s+1}^{p} \Gamma\left(1-b_{j}-t\right)} x^{-t} d t,
$$

where $i=\sqrt{-1}$ is the complex unit and $L$ denotes an integration path (Gradshteyn and Ryzhik, 2000, Section 9.3). The Meijer G-function contains as particular cases many integrals with elementary and special functions (Prudnikov et al., 1986). Further, we assume that $c=s / k$, where $s$ and $k$ are positive integers. This condition is not restrictive since every positive real number can be approximated by a rational number.

For $s$ and $k$ positive integers, $\mu>-1$ and $p>0$, the following result holds (Prudnikov et al., 1992, p. 21)

$$
\begin{aligned}
I\left(p, \mu, \frac{s}{k}, v\right) & =\int_{0}^{\infty} \mathrm{e}^{-p x} x^{\mu}\left(1+x^{\frac{s}{k}}\right)^{v} d x \\
& =V G_{k+s, k}^{k, k+s}\left(\left.\frac{s^{s}}{p^{s}}\right|_{\left(\begin{array}{c} 
\\
(s,-\mu), \Delta(k, v+1) \\
\Delta(k, 0)
\end{array}\right),}\right.
\end{aligned}
$$

where $V=\frac{k^{-v} s^{\mu+\frac{1}{2}}}{(2 \pi)^{\frac{s-1}{2}} \Gamma(-v) p^{\mu+1}}$ and $\Delta(k, a)=\frac{a}{k}, \frac{a+1}{k}, \ldots, \frac{a+k}{k}$. For $t<0$, we can write

$$
M_{Z}(t)=s I\left(-t, \frac{s}{k}-1, \frac{s}{k},-k-1\right) \text {. }
$$

Finally, the mgf of $X$ can be reduced to

$$
M_{X}(t)=s \sum_{m=0}^{\infty} b_{m} I\left(-t, \frac{s}{k(m+1)}-1, \frac{s}{k(m+1)},-k(m+1)-1\right) .
$$

\subsection{Residual and Reversed Residual Lifes}

For $n=1,2, \ldots$ and $t>0$, the $n$th moment of the residual life of $X$ is given by

$$
m_{n}(t)=\frac{1}{1-F(t)} \int_{t}^{\infty}(x-t)^{n} d F(x) .
$$


Using equation (9), we can write

$$
m_{n}(t)=\frac{k}{\bar{F}(t)} \sum_{m=0}^{\infty} \sum_{i=0}^{n} \frac{(n+1)_{i}(m+1) b_{m} t^{n-i}}{(-1)^{n-i} i !} B\left(t^{c} ; k(m+1)-\frac{i}{c}, \frac{i}{c}+1\right),
$$

where $\rho_{i}=\Gamma(\rho+1) / \Gamma(\rho-i+1)$ is the falling factorial.

The mean residual life (MRL) function of $X$ follows by setting $n=1$ in the last equation. It represents the expected additional life length for a unit which is alive at age $x$. The MRL is also known as the life expectancy at age $x$.

For $n=1,2, \ldots$ and $t>0$, the $n$th moment of the reversed residual life of $X$ is given by

$$
M_{n}(t)=\frac{1}{F(t)} \int_{0}^{t}(t-x)^{n} d F(x) \text {. }
$$

Then, we can write

$$
M_{n}(t)=\frac{k}{F(t)} \sum_{i=0}^{n} \sum_{m=0}^{\infty} \frac{(n+1)_{i}(m+1) b_{m} t^{n-i}}{(-1)^{i} i !} B\left(t^{c} ; k(m+1)-\frac{i}{c}, \frac{i}{c}+1\right) .
$$

The mean inactivity time (MIT) of $X$ follows by setting $n=1$ in the above equation. It represents the waiting time elapsed since the failure of an item on condition that this failure had occurred in $(0, x)$. The MIT is also called the mean reversed residual life function.

\subsection{Order Statistics}

Let $X_{1}, \ldots, X_{n}$ be a random sample of size $n$ from the MOEBXII distribution and $X_{(1)}, \ldots, X_{(n)}$ be the corresponding order statistics. Then, the pdf of the $i$ th order statistic $X_{i: n}$, say $f_{i: n}(x)$, is given by

$$
f_{i: n}(x)=\frac{f(x)}{\mathrm{B}(i, n-i+1)} \sum_{j=0}^{n-i}(-1)^{j}\left(\begin{array}{c}
n-i \\
j
\end{array}\right) F(x)^{i+j-1} .
$$

Using the generalized binomial expansion and after some algebra, we can write

$$
\begin{aligned}
& f(x) F(x)^{i+j-1}=\lambda \sum_{l, w, r=0}^{\infty}(-1)^{l+r} \alpha^{l+1} \bar{\alpha}^{w}\left(\begin{array}{c}
-l-2 \\
w
\end{array}\right)\left(\begin{array}{c}
l+w \\
r
\end{array}\right) \\
& \quad \times\left(\begin{array}{c}
i+j-1 \\
l
\end{array}\right) \text { c } k x^{c-1}\left(1+x^{c}\right)^{-k-1}\left[1-\left(1+x^{c}\right)^{-k}\right]^{\lambda(r+1)-1} .
\end{aligned}
$$

Using this expansion again, the last equation reduces to

$$
\begin{array}{r}
f(x) F(x)^{i+j-1}=\lambda \sum_{l, w, r, s=0}^{\infty}(-1)^{l+r+s} \alpha^{l+1} \bar{\alpha}^{w}\left(\begin{array}{c}
-l-2 \\
w
\end{array}\right)\left(\begin{array}{c}
l+w \\
r
\end{array}\right) \\
\times\left(\begin{array}{c}
i+j-1 \\
l
\end{array}\right)\left(\begin{array}{c}
\lambda(r+1)-1 \\
s
\end{array}\right) c k x^{c-1}\left(1+x^{c}\right)^{-k(s+1)-1} .
\end{array}
$$


Then, we can write

$$
f(x) F(x)^{i+j-1}=\sum_{s=0}^{\infty} p_{s} g_{s+1}(x),
$$

where

$$
\begin{aligned}
p_{s}= & \frac{\lambda}{s+1} \sum_{l, w, r=0}^{\infty}(-1)^{l+r+s} \alpha^{l+1} \bar{\alpha}^{w}\left(\begin{array}{c}
-l-2 \\
w
\end{array}\right)\left(\begin{array}{c}
l+w \\
r
\end{array}\right) \\
& \times\left(\begin{array}{c}
i+j-1 \\
l
\end{array}\right)\left(\begin{array}{c}
\lambda(r+1)-1 \\
s
\end{array}\right)
\end{aligned}
$$

and $g_{s+1}(x)$ denotes the BXII density function with parameters $c$ and $(s+1) k$.

By inserting (15) in equation (14), we obtain

$$
f_{i: n}(x)=\frac{1}{\mathrm{~B}(i, n-i+1)} \sum_{j=0}^{n-i}(-1)^{j}\left(\begin{array}{c}
n-i \\
j
\end{array}\right) \sum_{s=0}^{\infty} p_{s} g_{s+1}(x) .
$$

Equation (16) reveals that the density function of the MOEBXII order statistics is a linear combination of BXII densities. Based on this equation, we can obtain some structural properties of $X_{i: n}$ from those BXII properties. For example, the $q$ th moment of $X_{i: n}$ is given by

$$
E\left(X_{i: n}^{q}\right)=\frac{1}{\mathrm{~B}(i, n-i+1)} \sum_{j=0}^{n-i}(-1)^{j}\left(\begin{array}{c}
n-i \\
j
\end{array}\right) \sum_{s=0}^{\infty} p_{s} k(s+1) B\left(k(s+1)-\frac{q}{c}, \frac{q}{c}+1\right) .
$$

Using the moments in the last equation, we can derive explicit expressions for the Lmoments of $X$ as infinite weighted linear combinations of the means of suitable MOEBXII distributions. They are given by

$$
\lambda_{r}=\frac{1}{r} \sum_{d=0}^{r-1}(-1)^{d}\left(\begin{array}{c}
r-1 \\
d
\end{array}\right) E\left(X_{r-d: r}\right), r \geq 1 .
$$

\subsection{Probability Weighted Moments}

The PWMs can be used to derive estimators of the parameters and quantiles of generalized distributions. These moments have low variances and no severe biases, and they compare favorably with estimators obtained by maximum likelihood. The $(r, j)$ th PWM of $X$ (for $r \geq 1, j \geq 0$ ) is formally defined by

$$
\rho_{r, j}=E\left[X^{r} F(X)^{j}\right]=\int_{0}^{\infty} x^{r} F(x)^{j} f(x) d x .
$$

We can write from (9)

$$
\begin{aligned}
& f(x) F(x)^{j}=\lambda \sum_{l, w, r, s=0}^{\infty}(-1)^{l+r+s} \alpha^{l+1} \bar{\alpha}^{w}\left(\begin{array}{l}
j \\
l
\end{array}\right)\left(\begin{array}{c}
-l-2 \\
w
\end{array}\right)\left(\begin{array}{c}
l+w \\
r
\end{array}\right) \\
& \times\left(\begin{array}{c}
\lambda(r+1)-1 \\
s
\end{array}\right) c k x^{c-1}\left(1+x^{c}\right)^{-k(s+1)-1} \text {. }
\end{aligned}
$$

We can rewrite the last equation as

$$
f(x) F(x)^{j}=\sum_{s=0}^{\infty} d_{s} g_{s+1}(x)
$$


where

$$
d_{s}=\frac{\lambda}{s+1} \sum_{l, w, r=0}^{\infty}(-1)^{l+r+s} \alpha^{l+1} \bar{\alpha}^{w}\left(\begin{array}{l}
j \\
l
\end{array}\right)\left(\begin{array}{c}
-l-2 \\
w
\end{array}\right)\left(\begin{array}{c}
l+w \\
r
\end{array}\right)\left(\begin{array}{c}
\lambda(r+1)-1 \\
s
\end{array}\right) .
$$

Then, $\rho_{r, j}$ can be expressed as

$$
\rho_{r, j}=\sum_{s=0}^{\infty} d_{s} \int_{0}^{\infty} x^{r} g_{s+1}(x) d x
$$

Finally, we obtain (for $r<c k$ )

$$
\rho_{r, j}=\sum_{s=0}^{\infty} d_{s} k(s+1) B\left(k(s+1)-\frac{r}{c}, \frac{r}{c}+1\right) .
$$

\section{Estimation}

In this section, we consider the estimation of the unknown parameters of the MOEBXII model from complete samples by maximum likelihood and least square methods. The MLEs enjoy desirable properties for constructing confidence intervals.

Let $x_{1}, \ldots, x_{n}$ be a random sample of this distribution with parameter vector $\theta=$ $(c, k, \lambda, \alpha)^{\mathrm{T}}$. The log-likelihood function for $\theta$, say $\ell=\ell(\theta)$, is given by

$$
\begin{gathered}
\ell=n \log \alpha+n \log \lambda+n \log c+n \log k+(c-1) \sum_{i=1}^{n} \log x_{i}+(\lambda-1) \sum_{i=1}^{n} \log s_{i} \\
-(k+1) \sum_{i=1}^{n} \log \left(1+x_{i}^{c}\right)-2 \sum_{i=1}^{n} \log \left[1-\bar{\alpha}\left(1-s_{i}^{\lambda}\right)\right]
\end{gathered}
$$

where $s_{i}=\left[1-\left(1+x_{i}^{c}\right)^{-k}\right]$.

Equation (18) can be maximized either directly by using the R (optim function), SAS (PROC NLMIXED), Ox program (sub-routine MaxBFGS) or by solving the nonlinear likelihood equations obtained by differentiating this equation.

The elements of the score vector $\mathbf{U}(\theta)=\frac{\partial \ell}{\partial \theta}=\left(\frac{\partial \ell}{\partial c}, \frac{\partial \ell}{\partial k}, \frac{\partial \ell}{\partial \lambda}, \frac{\partial \ell}{\partial \alpha}\right)^{\mathrm{T}}$ are:

$$
\begin{gathered}
\frac{\partial \ell}{\partial c}=\frac{n}{c}+\sum_{i=1}^{n} \log x_{i}+(k+1) \sum_{i=1}^{n} \frac{x_{i}^{c} \log x_{i}}{1+x_{i}^{c}}+(\lambda-1) \sum_{i=1}^{n} \frac{p_{i}}{s_{i}}-2 \sum_{i=1}^{n} \frac{\bar{\alpha} \lambda p_{i}\left(s_{i}\right)^{\lambda-1}}{1-\bar{\alpha}\left(1-s_{i}^{\lambda}\right)^{\prime}}, \\
\quad \frac{\partial \ell}{\partial k}=\frac{n}{k}-\sum_{i=1}^{n} \log \left(1+x_{i}^{c}\right)+(\lambda-1) \sum_{i=1}^{n} \frac{u_{i}}{s_{i}}-2 \bar{\alpha} \lambda \sum_{i=1}^{n} \frac{u_{i} s_{i}^{\lambda-1}}{1-\bar{\alpha}\left(1-s_{i}^{\lambda}\right)^{\prime}} \\
\frac{\partial \ell}{\partial \lambda}=\frac{n}{\lambda}+\sum_{i=1}^{n} \log s_{i}-2 \sum_{i=1}^{n} \frac{\bar{\alpha} s_{i}^{\lambda} \log s_{i}}{1-\bar{\alpha}\left(1-s_{i}^{\lambda}\right)} \text { and } \frac{\partial \ell}{\partial \alpha}=\frac{n}{\alpha}-2 \sum_{i=1}^{n} \frac{\left(1-s_{i}^{\lambda}\right)}{1-\bar{\alpha}\left(1-s_{i}^{\lambda}\right)^{\prime}}
\end{gathered}
$$

respectively, where $p_{i}=k x_{i}^{c}\left(1+x_{i}^{c}\right)^{-k-1} \log \left(x_{i}\right)$ and $u_{i}=\left(1+x_{i}^{c}\right)^{-k} \log \left(1+x_{i}^{c}\right)$. 
We can obtain the estimates of the unknown parameters by setting the score vector to zero, $\mathbf{U}(\hat{\theta})=\mathbf{0}$. By solving these equations simultaneously gives the MLEs $\hat{c}, \hat{k}, \hat{\lambda}$ and $\hat{\alpha}$. Iterative techniques such as Newton-Raphson type algorithms can be adopted to obtain the estimates. All the second-order derivatives exist for the proposed distribution.

The interval estimation of the model parameters requires the $4 \times 4$ observed information matrix $J(\theta)=\left\{J_{i j}\right\}$ for $i, j=c, k, \lambda, \alpha$. The multivariate normal $N_{4}\left(0, J(\hat{\theta})^{-1}\right)$ distribution, under standard regularity conditions, can be used to provide approximate confidence intervals for the unknown parameters, where $J(\hat{\theta})$ is the total observed information matrix evaluated at $\hat{\theta}$. Then, approximate $100(1-\varphi) \%$ confidence intervals for $c, k, \lambda$ and $\alpha$ are given by

$$
\hat{c} \pm z_{\varphi / 2} \sqrt{\hat{J}_{c c}}, \quad \hat{k} \pm z_{\varphi / 2} \sqrt{\hat{J}_{k k}}, \hat{\lambda} \pm z_{\varphi / 2} \sqrt{\hat{J}_{\lambda \lambda}} \text { and } \hat{\alpha} \pm z_{\varphi / 2} \sqrt{\hat{J}_{\alpha \alpha}},
$$

where $z_{\varphi / 2}$ is the upper $\varphi$ th percentile of the standard normal model.

An alternative estimation to maximum likelihood is the least square estimation. The least square estimates (LSEs), $\tilde{c}, \tilde{k}, \tilde{\lambda}$ and $\tilde{\alpha}$ of $c, k, \lambda$ and $\alpha$, are defined as those arguments that minimize the objective function:

$$
Q(\theta)=\sum_{i=1}^{n}\left(1-\frac{\alpha\left\{1-\left[1-\left(1+x_{(i)}^{c}\right)^{-k}\right]^{\lambda}\right\}}{1-\bar{\alpha}\left\{1-\left[1-\left(1+x_{(i)}^{c}\right)^{-k}\right]^{\lambda}\right\}}-\frac{i}{n+1}\right)^{2},
$$

where $x_{(i)}$ is a possible outcome of the $i$ th order statistic based on a $n$-points random sample obtained from $X$. For more complex distributions, the properties of the LSEs are not easy to obtain as those of the MLEs.

The minimum point $\tilde{c}, \tilde{k}, \tilde{\lambda}$ and $\tilde{\alpha}$ can also be given as a solution in the following system of non-linear equations:

$$
\frac{\partial Q(\theta)}{\partial c}=\frac{\partial Q(\theta)}{\partial k}=\frac{\partial Q(\theta)}{\partial \lambda}=\frac{\partial Q(\theta)}{\partial \alpha}=0
$$

\section{Simulation Study}

In this section, we provide simulation results to assess the performance of the MLEs and LSEs of. We simulate the MOEBXII model under two setups for the model parameters: $c=1.5, k=2.0, \lambda=1.5$ and $\alpha=0.5$; and $c=3.0, k=0.5, \lambda=1.5$ and $\alpha=5.0$.

The results are obtained from 3,000 Monte Carlo simulations. For each replication, a random sample of size $n$ is drawn from the MOEBXII model and the parameters are estimated by MLEs. The sample sizes are $n=50,100,200,300$ and 500. All simulations were performed using the $\mathbf{R}$ software with the Optim function.

Table 2 lists the averages of the MLEs (Mean), the biases (Bias) and the mean square errors (MSEs) and 95\% coverage probabilities (CP). We conclude from the figures in Table 2 that the MSEs of the MLEs of $c, k, \lambda$ and $\alpha$ decay toward zero when the sample size increases, as expected under standard asymptotic theory. In fact, the biases of the estimates tend to be closer to the true parameter values if $n$ increases. 
Table 3 presents the averages of the LSEs (Mean), the biases (Bias) and the mean square errors (MSEs). We conclude from the figures in Table 3 that the biases and MSEs of the LSEs of $c, k, \lambda$ and $\alpha$ are, in general, lower than those values for the MLEs.

Table 2: Summaries of the MLEs for the MOEBXII model

\begin{tabular}{|c|c|c|c|c|c|c|c|c|c|}
\hline & & $c=1.5$ & $k=2.0$ & $\lambda=1.5$ & $\alpha=0.5$ & $c=3.0$ & $k=0.5$ & $\lambda=1.5$ & $\alpha=5.0$ \\
\hline$n$ & & Mean & Bias & MSE & $\mathrm{CP}$ & Mean & Bias & MSE & $\mathrm{CP}$ \\
\hline \multirow{4}{*}{50} & $c$ & 1.6787 & 0.1787 & 0.3923 & 0.9793 & 3.5450 & 0.5450 & 4.1481 & 0.9917 \\
\hline & $k$ & 2.1787 & 0.1787 & 1.1016 & 0.9653 & 0.5264 & 0.0264 & 0.1068 & 0.9253 \\
\hline & $\lambda$ & 1.6037 & 0.1037 & 0.7354 & 0.9577 & 2.1481 & 0.6481 & 1.4024 & 0.9823 \\
\hline & $\alpha$ & 0.7814 & 0.2814 & 0.5862 & 0.9420 & 4.0536 & -0.9464 & 7.7797 & 0.8730 \\
\hline \multirow{4}{*}{100} & $c$ & 1.6127 & 0.1127 & 0.2504 & 0.9840 & 3.2913 & 0.2913 & 2.2502 & 0.9973 \\
\hline & $k$ & 2.1280 & 0.1280 & 0.9799 & 0.9723 & 0.5272 & 0.0272 & 0.0569 & 0.9347 \\
\hline & $\lambda$ & 1.6403 & 0.1403 & 0.6092 & 0.9710 & 1.9946 & 0.4946 & 0.8754 & 0.9807 \\
\hline & $\alpha$ & 0.6595 & 0.1595 & 0.2975 & 0.9253 & 4.2978 & -0.7022 & 6.3629 & 0.9160 \\
\hline \multirow{4}{*}{200} & $c$ & 1.6085 & 0.1085 & 0.1979 & 0.9887 & 3.0533 & 0.0533 & 1.2754 & 0.9937 \\
\hline & $k$ & 2.0052 & 0.0052 & 0.6845 & 0.9683 & 0.5538 & 0.0538 & 0.0450 & 0.9533 \\
\hline & $\lambda$ & 1.6018 & 0.1018 & 0.4408 & 0.9740 & 1.8746 & 0.3746 & 0.6255 & 0.9743 \\
\hline & $\alpha$ & 0.5443 & 0.0443 & 0.1103 & 0.9237 & 4.6238 & -0.3762 & 4.6908 & 0.9523 \\
\hline \multirow{4}{*}{300} & $c$ & 1.6124 & 0.1124 & 0.1699 & 0.9883 & 3.1017 & 0.1017 & 1.0557 & 0.9940 \\
\hline & $k$ & 1.9544 & -0.0456 & 0.5453 & 0.9660 & 0.5335 & 0.0335 & 0.0332 & 0.9520 \\
\hline & $\lambda$ & 1.5488 & 0.0488 & 0.3306 & 0.9693 & 1.7611 & 0.2611 & 0.4615 & 0.9670 \\
\hline & $\alpha$ & 0.5189 & 0.0189 & 0.0714 & 0.9263 & 4.8136 & -0.1864 & 4.0601 & 0.9717 \\
\hline \multirow{4}{*}{500} & $c$ & 1.6016 & 0.1016 & 0.1332 & 0.9897 & 3.0977 & 0.0977 & 0.6927 & 0.9790 \\
\hline & $k$ & 1.9327 & -0.0673 & 0.3892 & 0.9653 & 0.5154 & 0.0154 & 0.0193 & 0.9423 \\
\hline & $\lambda$ & 1.5149 & 0.0149 & 0.2225 & 0.9670 & 1.6412 & 0.1412 & 0.2439 & 0.9510 \\
\hline & $\alpha$ & 0.5011 & 0.0011 & 0.0394 & 0.9297 & 4.9565 & -0.0435 & 2.8801 & 0.9653 \\
\hline
\end{tabular}

Table 3: Summaries of the LSEs for the MOEBXII model

\begin{tabular}{lllllllll}
\hline \hline$n$ & & Mean & Bias & MSE & & Mean & Bias & MSE \\
\hline \multirow{2}{*}{50} & $c=1.5$ & 1.4621 & -0.0379 & 0.1450 & $c=3$ & 3.733 & 0.7330 & 6.5609 \\
& $k=2$ & 1.8469 & -0.1531 & 0.5194 & $k=0.5$ & 0.5743 & 0.0743 & 0.1398 \\
& $\lambda=1.5$ & 1.6906 & 0.1906 & 0.6319 & $\lambda=2$ & 1.6021 & 0.1021 & 0.5461 \\
& $\alpha=0.5$ & 0.7786 & 0.2786 & 0.8705 & $\alpha=5$ & 4.7225 & -0.2775 & 1.8995 \\
\hline \multirow{3}{*}{100} & $c=1.5$ & 1.4762 & -0.0238 & 0.0837 & $c=3$ & 3.3904 & 0.3904 & 3.5382 \\
& $k=2$ & 1.8934 & -0.1066 & 0.3284 & $k=0.5$ & 0.5741 & 0.0741 & 0.0937 \\
& $\lambda=1.5$ & 1.6519 & 0.1519 & 0.4228 & $\lambda=2$ & 1.5884 & 0.0884 & 0.2909 \\
& $\alpha=0.5$ & 0.6310 & 0.1310 & 0.3838 & $\alpha=5$ & 4.8605 & -0.1395 & 0.8140 \\
\hline \multirow{3}{*}{200} & $c=1.5$ & 1.4762 & -0.0238 & 0.0491 & $c=3$ & 3.1389 & 0.1389 & 1.7273 \\
& $k=2$ & 1.8792 & -0.1208 & 0.1906 & $k=0.5$ & 0.5692 & 0.0692 & 0.0578 \\
& $\lambda=1.5$ & 1.6427 & 0.1427 & 0.2475 & $\lambda=2$ & 1.5642 & 0.0642 & 0.1375 \\
& $\alpha=0.5$ & 0.5019 & 0.0019 & 0.1318 & $\alpha=5$ & 4.9543 & -0.0457 & 0.4205 \\
\hline \multirow{3}{*}{300} & $c=1.5$ & 1.4849 & -0.0151 & 0.0356 & $c=3$ & 3.0783 & 0.0783 & 1.0371 \\
& $k=2$ & 1.9007 & -0.0993 & 0.1317 & $k=0.5$ & 0.5554 & 0.0554 & 0.0401 \\
& $\lambda=1.5$ & 1.6075 & 0.1075 & 0.1541 & $\lambda=2$ & 1.5439 & 0.0439 & 0.0871 \\
& $\alpha=0.5$ & 0.4744 & -0.0256 & 0.0643 & $\alpha=5$ & 4.9831 & -0.0169 & 0.2901 \\
\hline \multirow{2}{*}{500} & $c=1.5$ & 1.5012 & 0.0012 & 0.0243 & $c=3$ & 3.0438 & 0.0438 & 0.6100 \\
& $k=2$ & 1.9244 & -0.0756 & 0.0873 & $k=0.5$ & 0.5382 & 0.0382 & 0.0245 \\
& $\lambda=1.5$ & 1.5541 & 0.0541 & 0.0871 & $\lambda=2$ & 1.525 & 0.0250 & 0.0485 \\
& $\alpha=0.5$ & 0.4765 & -0.0235 & 0.0290 & $\alpha=5$ & 4.9865 & -0.0135 & 0.1797 \\
\hline \hline
\end{tabular}




\section{Applications}

In this section, we provide two applications of the new distribution to prove empirically its potentiality. We shall compare the fits of the MOEBXII model and the following competing non-nested distributions, namely: the Weibull Lomax (WL) (Tahir et al., 2015), beta generalized exponential (BGE) (Cordeiro et al., 2010), beta Burr XII (BBXII) (Cordeiro et al., 2010), beta Weibull (BW) (Lee et al., 2007), Kumaraswamy Lomax (KwL) (Lemonte and Cordeiro, 2013) and Kumaraswamy log-logistic (KwLL) (de Santana et al., 2012) distributions, whose pdfs (for $x>0$ ) are given below:

$\mathrm{WL}: f(x)=\frac{c k \lambda}{\alpha}\left(1+\frac{x}{\alpha}\right)^{c k-1}\left[1-\left(1+\frac{x}{\alpha}\right)^{-k}\right]^{c-1} e^{-\lambda\left[\left(1+\frac{x}{\alpha}\right)^{k}-1\right]^{c}}$;

BGE: $f(x)=\frac{c k}{B(\alpha, \lambda)} e^{-c x}\left(1-e^{-c x}\right)^{k \alpha-1}\left[1-\left(1-e^{-c x}\right)^{k}\right]^{\lambda-1}$;

BBXII: $f(x)=\frac{c k}{B(\alpha, \lambda)} x^{c-1}\left(1+x^{c}\right)^{-k \lambda-1}\left[1-\left(1+x^{c}\right)^{-k}\right]^{\alpha-1}$;

$\mathrm{BW}: f(x)=\frac{c x^{c-1}}{k^{c} B(\alpha, \lambda)} e^{-\lambda(x / k)^{c}}\left[1-e^{-(x / k)^{c}}\right]^{\alpha-1}$;

$\mathrm{KwL}: f(x)=\frac{c k \lambda}{\alpha}\left(1+\frac{x}{\alpha}\right)^{-k-1}\left[1-\left(1+\frac{x}{\alpha}\right)^{-k}\right]^{c-1} \times\left\{1-\left[1-\left(1+\frac{x}{\alpha}\right)^{-k}\right]^{c}\right\}^{\lambda-1}$

KwLL: $f(x)=\frac{c k \lambda}{\alpha^{c k}} x^{c k-1}\left[1+\left(\frac{x}{\alpha}\right)^{c}\right]^{-k-1}\left(1-\left\{1-\left[1+\left(\frac{x}{\alpha}\right)^{c}\right]^{-1}\right\}^{k}\right)^{\lambda-1}$.

The parameters of the above densities are all positive real numbers.

In order to compare these distributions, we consider some goodness-of-fit measures including $-2 \hat{\ell}$ (maximized log-likelihood), AIC (Akaike information criterion), BIC (Bayesian information criterion), CAIC (consistent Akaike information criterion), Kolmogorov-Smirnov (K-S) statistic and $p$-value.

The first data set refers to the remission times (in months) of a random sample of 128 bladder cancer patients (Lee and Wang, 2003). These data were previously analyzed by Cordeiro et al. (2012) and Nofal et al. (2017).

The second uncensored data set consists of 20 observations. These data are reported in Gross and Clark (1975, p. 105) on the relief times of twenty patients receiving an analgesic and analyzed by Mead (2016) and Afify et al. (2017) to fit the beta exponentiated Lomax and beta transmuted Lindley distributions, respectively.

Table 4: Goodness-of-fit statistics for cancer data

\begin{tabular}{lcccccc}
\hline \hline Model & $-2 \hat{\ell}$ & $A I C$ & $B I C$ & $C A I C$ & K-S & p-value \\
\hline MOEBXII & 818.979 & 826.979 & 838.387 & 827.304 & 0.02655 & 0.99999 \\
KwL & 819.873 & 827.873 & 839.281 & 828.198 & 0.0332 & 0.99894 \\
WL & 820.148 & 828.148 & 839.556 & 828.473 & 0.03575 & 0.99672 \\
BW & 821.401 & 829.401 & 840.809 & 829.726 & 0.04082 & 0.9833 \\
KwLL & 821.814 & 829.814 & 841.223 & 830.14 & 0.04348 & 0.96891 \\
BGE & 826.595 & 834.595 & 846.003 & 834.92 & 0.07338 & 0.49584 \\
BBXII & 831.471 & 839.471 & 850.879 & 839.796 & 0.05747 & 0.79165 \\
\hline \hline
\end{tabular}


Table 5: MLEs and their standard errors (in parentheses) for cancer data

\begin{tabular}{ccccc}
\hline \hline Model & $c$ & $k$ & $\lambda$ & $\alpha$ \\
\hline MOBXII & 0.5051 & 4.8881 & 4.2618 & 110.4832 \\
& $(0.0022)$ & $(0.3310)$ & $(2.151)$ & $(1.1000)$ \\
\hline KwL & 1.5157 & 0.3903 & 12.0549 & 12.2950 \\
& $(0.2660)$ & $(1.2280)$ & $(45.0160)$ & $(11.7540)$ \\
\hline WL & 1.5152 & 0.1102 & 36.1153 & 6.9692 \\
& $(0.2870)$ & $(0.1810)$ & $(109.9380)$ & $(6.9280)$ \\
\hline \multirow{2}{*}{ BW } & 0.6290 & 4.3830 & 1.3360 & 2.9110 \\
& $(0.560)$ & $(22.8250)$ & $(7.5220)$ & $(3.3390)$ \\
\hline KwLL & 0.2360 & 12.6680 & 252.4910 & 0.6960 \\
& $(0.1300)$ & $(2.5330)$ & $(719.2900)$ & $(2.5400)$ \\
\hline BGE & 0.0816 & 1.1887 & 1.49851 & 0.9893 \\
& $(0.0450)$ & $(0.3650)$ & $(0.8200)$ & $(0.4230)$ \\
\hline BBXII & 0.2840 & 0.7760 & 24.5330 & 27.5430 \\
& $(0.1590)$ & $(0.7220)$ & $(24.3560)$ & $(27.7270)$ \\
\hline \hline
\end{tabular}

Table 6: Goodness-of-fit statistics for relief times data

\begin{tabular}{lcccccc}
\hline \hline Model & $-2 \hat{\ell}$ & AIC & BIC & CAIC & K-S & p-value \\
\hline \hline MOEBXII & 30.792 & 38.792 & 42.774 & 41.458 & 0.08432 & 0.99887 \\
KwLL & 30.822 & 38.822 & 42.805 & 41.489 & 0.08508 & 0.99869 \\
BBXII & 30.845 & 38.845 & 42.828 & 41.512 & 0.08577 & 0.99851 \\
BW & 34.453 & 42.453 & 46.436 & 45.120 & 0.16118 & 0.67636 \\
BGE & 35.329 & 43.329 & 47.312 & 45.995 & 0.17054 & 0.60585 \\
KwL & 36.75 & 44.75 & 48.733 & 47.416 & 0.17464 & 0.5753 \\
WL & 41.393 & 49.393 & 53.375 & 52.059 & 0.18387 & 0.50834 \\
\hline \hline
\end{tabular}

Tables 4 and 6 list the values of $-2 \hat{\ell}, A I C, B I C, C A I C, \mathrm{~K}-\mathrm{S}$ and $p$-value, whereas the MLEs and their corresponding standard errors (in parentheses) of the model parameters are given in Tables 5 and 7, for the two data sets, respectively.

The fitted pdfs of the MOEBXII, KwL, WL and BW models and QQ-plots for cancer data are displayed in Figure 5, whereas Figure 6 displays the estimated cdfs and survival functions of these fitted models. 
Table 7: MLEs and their standard errors (in parentheses) for relief times data

\begin{tabular}{lcccc}
\hline \hline Model & $c$ & $k$ & $\lambda$ & $\alpha$ \\
\hline MOBXII & 0.8625 & 6.3711 & 509.9555 & 0.4456 \\
& $(2.5200)$ & $(24.7590)$ & $(8736)$ & $(1.4462)$ \\
\hline KwLL & 4.1980 & 14.7800 & 0.9910 & 0.8320 \\
& $(3.8080)$ & $(89.7230)$ & $(1.2990)$ & $(1.4870)$ \\
\hline BBXII & 5.3690 & 0.7260 & 1.0650 & 6.0600 \\
& $(34.5050)$ & $(11.9590)$ & $(12.3380)$ & $(55.7400)$ \\
\hline BW & 0.6180 & 1.3670 & 12.7280 & 28.7650 \\
& $(0.3890)$ & $(1.9820)$ & $(22.4910)$ & $(41.1200)$ \\
\hline BGE & 0.2927 & 0.4216 & 13.2883 & 29.1788 \\
& $(0.5540)$ & $(1.1840)$ & $(27.8130)$ & $(93.7110)$ \\
\hline KwL & 27.9580 & 0.4610 & 466.9410 & 0.0630 \\
& $(68.1460)$ & $(0.1460)$ & $(808.7400)$ & $(0.2780)$ \\
\hline WL & 2.6957 & 16.7036 & 3443.6380 & 747.2166 \\
& $(0.4030)$ & $(141.7510)$ & $(15220)$ & $(64900)$ \\
\hline \hline
\end{tabular}

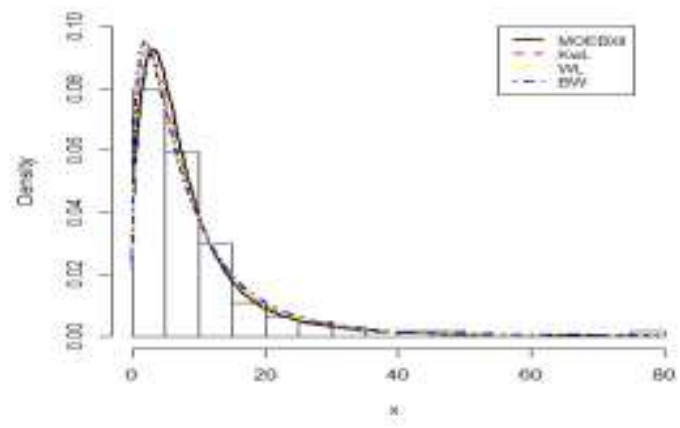

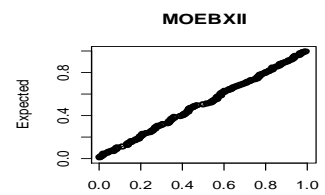

Observed

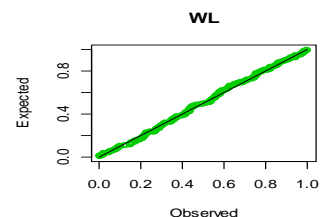

Observed

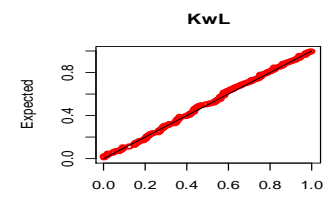

Observed

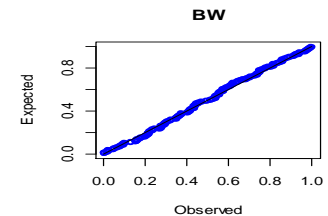

Figure 5: The estimated MOEBXII pdf and other estimated pdfs (left panel) and the QQplots of the MOEBXII, KwL, WL and BW models (right panel) for cancer data.

Figure 7 displays the fitted pdfs of the MOEBXII, KwLL, BBXII and BW distributions and QQ-plots for relief times data. Figure 8 gives the plots of the estimated cdfs and survival functions for the fitted models.

Overall, the values of the goodness-of-fit statistics in Tables 4 and 6 indicate that the MOEBXII model has the lowest values of these statistics among all fitted competitive models. Moreover, the plots in Figures 5, 6, 7 and 8 reveal that this model gives a close fit to both data sets. So, the MOEBXII distribution could be chosen as the best model to fit both data sets. 

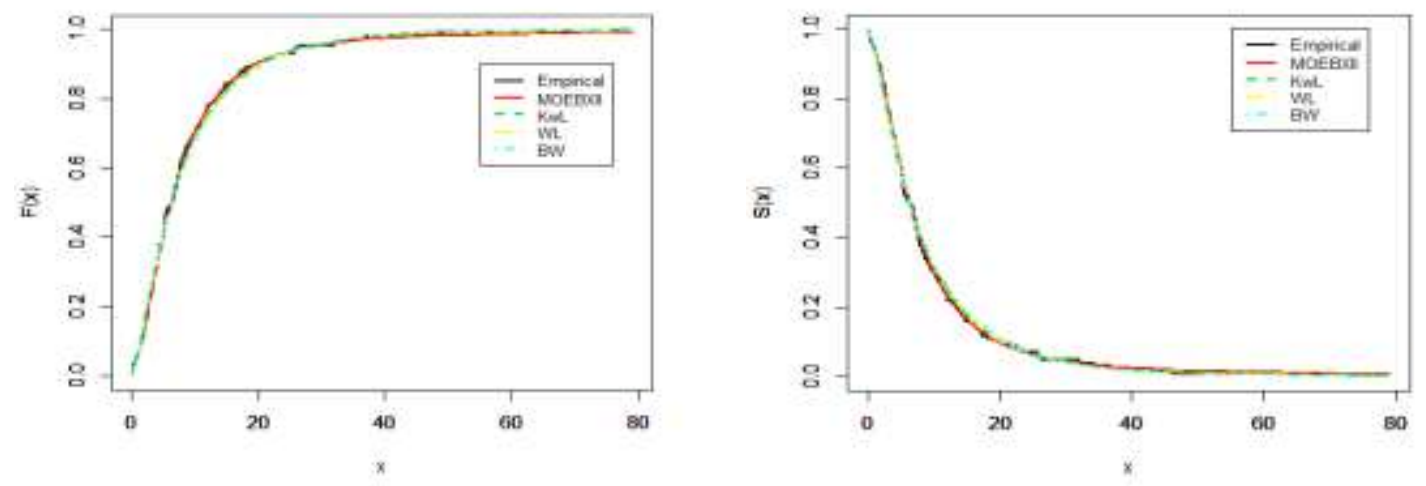

Figure 6: The estimated cdf of the MOEBXII model and other estimated cdfs (left panel) and estimated survival function of the MOEBXII model and other estimated survival functions (right panel) for cancer data.

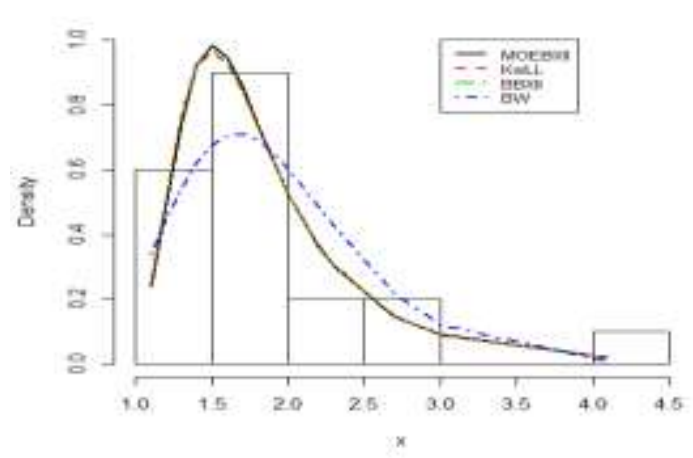

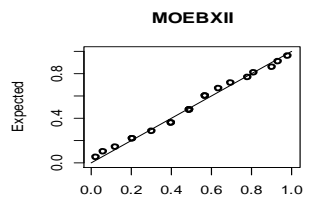

Observed

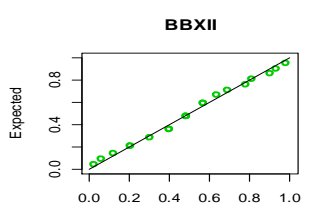

Observed

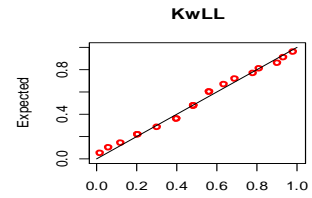

Observed

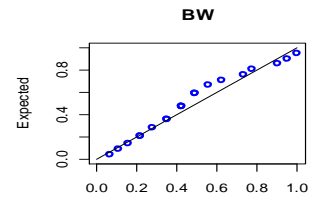

Observed

Figure 7: The estimated MOEBXII pdf and other estimated pdfs (left panel) and the QQplots of the MOEBXII, KwLL, BBXII and BW models (right panel) for relief times data.
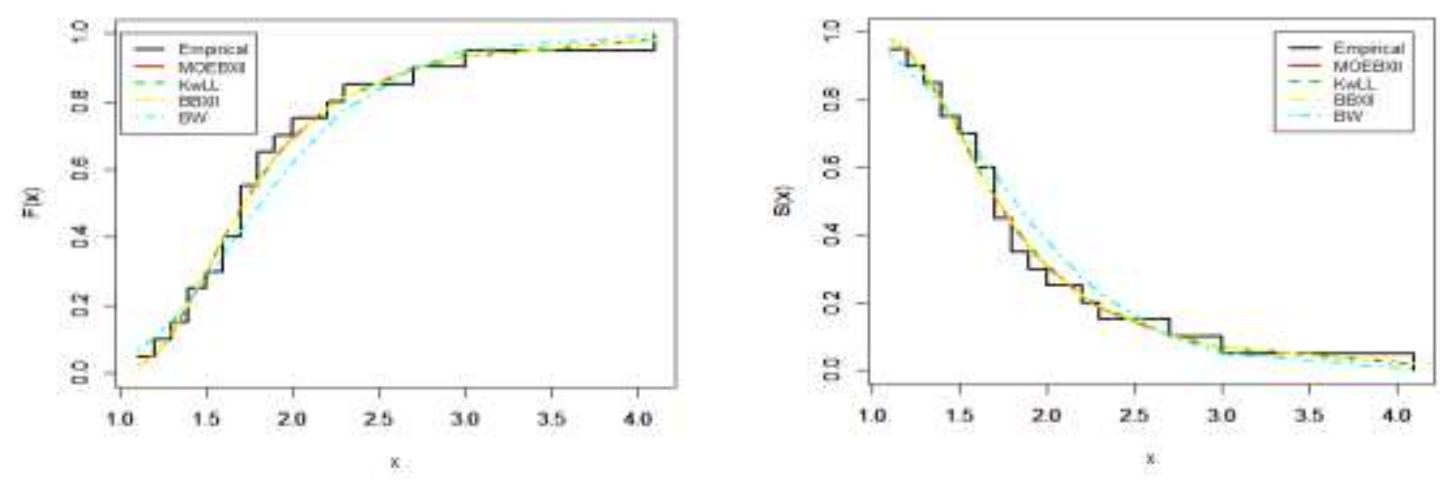

Figure 8: The estimated cdf of the MOEBXII model and other estimated cdfs (left panel) and estimated survival function of the MOEBXII model and other estimated survival functions (right panel) for relief times data. 


\section{Conclusions}

In this paper, we propose a new four-parameter model called the Marshall-Olkin exponentiated Burr XII (MOEBXII) distribution, which extends the exponentiated Burr XII (EBXII) distribution. The MOEBXII density function can be expressed as a linear combination of BXII densities. We derive explicit expressions for the ordinary and incomplete moments, quantile and generating functions, residual and reversed residual life functions. We also obtain the density function of the order statistics and their moments. We discuss maximum likelihood and least squares estimation. The proposed distribution provides better fits than some other nested and non-nested models using two real data sets. We hope that the proposed model will attract wider applications in areas such as survival and lifetime data, meteorology, hydrology, reliability, engineering and others

\section{References}

1. Afify, A. Z., Cordeiro, G. M., Ortega, E. M. M., Yousof, H. M. and Butt, N. S. (2016). The four-parameter Burr XII distribution: properties, regression model and applications. Communication in Statistics Theory-Methods, forthcoming.

2. Afify A. Z., Yousof, H. M. and Nadarajah, S. (2017). The beta transmuted-H family for lifetime data. Statistics and Its Interface, 10, 505-520.

3. Al-Saiari, A. Y., Baharith, L. A. and Mousa, S. A. (2014). Marshall-Olkin extended Burr type XII distribution. International Journal of Statistics and Probability, 3, 78-84.

4. Gomes, A. E., da-Silva, C. Q. and Cordeiro, G. M. (2015). Two extended Burr models: Theory and practice. Communication in Statistics Theory-Methods, 44, 1706-1734.

5. Barreto, M. L., Farenzena, G. G., Fiaccone, R. L., Santos, L. M. P., Assis, A. M. O., Araújo, M. P. N. and Santos, P. A. B. (1994). Effect of vitamin A supplementation on diarrhoea and acute lower-respiratory-tract infections in young children in Brazil. The Lancet, 344, 228-231.

6. Bourguignon, M., Silva, R. B. and Cordeiro, G. M. (2014). The Weibull-G family of probability distributions. Journal of Data Science, 12, 53-68.

7. Burr, I. W. (1942). Cumulative frequency functions. Annals of Mathematical Statistics, 13, 215-232.

8. Chen, G. and Balakrishnan, N. (1995). A general purpose approximate goodnessof-fit test. Journal of Quality Technology, 27, 154 -161.

9. Cordeiro, G. M., Lemonte, A. J. and Ortega, E. M. M. (2014). The MarshallOlkin Family of Distributions: Mathematical Properties and New Models. Journal of Statistical Theory and Practice, 8, 343-366.

10. Gradshteyn, I. S. and Ryzhik, I. M. (2000). Table of Integrals, Series and Products (sixth edition). San Diego: Academic Press.

11. Mead, M. E. (2014). The beta exponentiated Burr XII distribution. Journal of Statistics: Advances in Theory and Applications, 12, 53-73. 
12. Mead M. E. (2016). On five-parameter Lomax distribution: properties and applications. Pak. J. Statist. Oper. Res., 12, 185-199.

13. Mead, M. E. and Afify, A. Z. (2017). On five parameter Burr XII distribution: properties and applications. South African Statistical Journal, 51, 67-80.

14. Nofal, Z. M., Afify, A. Z., Yousof, H. M. and Cordeiro, G. M. (2017). The generalized transmuted-G family of distributions. Comm. Statist. Theory Methods, 46, 4119-4136.

15. Paranaíba, P. F., Ortega, E. M. M., Cordeiro, G. M. and Pescim, R. R. (2011). The beta Burr XII distribution with application to lifetime data. Computation Statistics and Data Analysis, 55, 1118-1136.

16. Paranaíba, P. F., Ortega, E. M. M., Cordeiro, G. M. and de Pascoa, M. (2013). The Kumaraswamy Burr XII distribution: theory and practice. Journal of Statistical Computation and Simulation, 83, 2117-2143.

17. Prudnikov, A. P., Brychkov, Y. A. and Marichev, O. I. (1986). Integrals and Series, 1. Gordon and Breach Science Publishers, Amsterdam.

18. Prudnikov, A. P., Brychkov, Y. A. and Marichev, O. I. (1992). Integrals and Series, 4. Gordon and Breach Science Publishers, Amsterdam.

19. Shao, Q. (2004). Notes on maximum likelihood estimation for the three-parameter Burr XII distribution . Computational Statistics and Data Analysis, 45, 675-687.

20. Tadikamalla, P. R. (1980). A look at the Burr and related distributions, International Statistical Review, 48, 337-344. 